\title{
Dynamical resonance quench and Fano interference in spontaneous Raman scattering from quasiparticle and collective excitations
}

\author{
J. Zhu, ${ }^{1}$ R. B. Versteeg, ${ }^{1}$ P. Padmanabhan, ${ }^{1}$ and P. H. M. van Loosdrecht ${ }^{1}$, ${ }^{\circ}$ \\ ${ }^{1}$ II. Physikalisches Institut, Universität zu Köln, Zülpicher Straße 77, D-50937 Köln, Germany
}

(Dated: February 8, 2019)

\begin{abstract}
Time-resolved spontaneous Raman spectroscopy serves as a probe for incoherent quasiparticle and collective excitation dynamics, and allows to distinguish symmetry changes across a photoinduced phase transition through the inelastic light scattering selection rules. Largely unexplored is the role of the Raman resonance enhancement in the time-domain, and the transient interaction between scattering from quasiparticles and collective excitations, with the latter interaction leading to a Fano interference.

In this work, we report on the observation of a phonon Raman tensor quench and Fano interference after strong photoexcitation of an intrinsic semiconductor. We observed a dynamic phonon scattering rate asymmetry and spectral asymmetry through simultaneous detection of both the antiStokes and Stokes response. The asymmetric phonon scattering rate is ascribed to the combined effect of the transient phonon population and the reduction of the phonon Raman tensor resulting from the photoexcited hole population. This same hole population results in a strong enhancement of the Fano spectral asymmetry.
\end{abstract}

Keywords: time-resolved spontaneous Raman spectroscopy, Raman tensor quench, dynamic Fanointerference

A quantum-mechanical description of the nature and transport properties of the various quasiparticles and collective excitations in semiconductors is integral to characterizing the physical properties of a wide array of technologically relevant materials. ${ }^{[12}$ Hot carrier and phonon relaxation dynamics in semiconductors,$\sqrt{314}$ for example, plays a central role in our understanding of carrier transport and energy dissipation in electronic and optoelectronic devices. As such, an accurate interpretation of the quasiparticle and collective excitation interactions and dynamics is vital not only from a fundamental perspective, but also to enable new applications.

Over the past four decades, a range of ultrafast spectroscopic probes have provided us insight into carrier and phonon relaxation dynamics and their respective timescales in semiconductors $\frac{415}{4}$ These measurements often rely on the coherent excitation of phonons, of which the dynamics is consecutively probed in the modulation of the refractive index $\left[\frac{6}{8} \mathrm{~A}\right.$ more direct view of phonon dynamics is provided by time-resolved inelastic scattering techniques. ${ }^{[12}$ This has the advantage over coherent excitation techniques that one can follow the true time evolution of a photoexcited material through its incoherent response, rather than the time evolution of coherent excitations.

Time-resolved spontaneous Raman scattering is a reasonably well-established probe for incohorent phonon population dynamics in semiconductors $\frac{11] 13}{18}$ and vibrational dynamics in molecules. ${ }^{1119 \sqrt[25]{25}}$ More recently the technique has been applied to study phonon dynamics in a range of carbon allotropes 112632 Through detailed balance of the anti-Stokes $\mathrm{I}_{\mathrm{AS}}$ to Stokes $\mathrm{I}_{\mathrm{S}}$ scattering intensity ratio the (phonon) population number $n$ can be directly quantified by $\mathrm{I}_{\mathrm{AS}} / \mathrm{I}_{\mathrm{S}} \propto n /(n+1)$. This relation stems from the fluctuation dissipation theorem,, 33 which on ultrafast timescales $(\ll \mathrm{ps})$ may lose its validity due to the presence of truly non-thermal occupation statistics. The inelastic light scattering selection rules $\left|\hat{\mathrm{E}}_{S} \cdot \chi_{A}^{\prime \prime} \cdot \hat{\mathrm{E}}_{\mathrm{I}}\right|^{2}$ for incoming electric field $\hat{\mathrm{E}}_{\mathrm{I}}$ and scattered field $\hat{\mathrm{E}}_{\mathrm{S}}$ entail the symmetry $A$ of the Raman-scattering channel $\chi_{A}^{\prime \prime}$. 34 This may be used to probe symmetry changes across a photoinduced phase transition, as was demonstrated for a high-to-low structural symmetry transition in antimony,$\frac{35}{35}$ and melting of the superconducting condensate in a high-temperature cuprate superconductor. ${ }^{36}$ Two largely unexplored aspects to time-resolved spontaneous Raman are the role of the Raman resonance enhancement in the time-domain,, 32 and the dynamic interaction between quasiparticle and collective excitation scattering, with the latter interaction leading to a Fano interference.

Here we demonstrate a photo-induced resonance quench, and Fano-interference in the transient phononic and electronic spontaneous Raman response of an intrinsic indirect band gap semiconductor. An intrinsic (100) oriented silicon sample (resistivity $>10000 \Omega \cdot \mathrm{cm}$ ) was used for the experiments. Time-resolved Raman scattering experiments were performed using a $740 \mathrm{~nm}$ optical pump pulse with a temporal width of $0.3 \mathrm{ps}$. The transient Raman spectra are recorded in a backscattering geometry using a $512 \mathrm{~nm}, 1.5 \mathrm{ps}$ probe pulse ${ }^{12}$ The polarization of both the pump and probe beams were aligned with the [110] crystallographic direction of the sample and the cross polarized scattered light was measured. The steady-state Raman spectrum of silicon is well-known, the main features being the optical phonon peak centered at $\pm 520 \mathrm{~cm}^{-1}$, and, in $p$-doped silicon, a broad and relatively weak electronic continuum extending to several hundred $\mathrm{cm}^{-1}$. This feature is attributed to inter-valence band Raman scattering between the uppermost heavy- and light-hole valence bands. ${ }^{[3740} \mathrm{Cou}-$ 
(a)

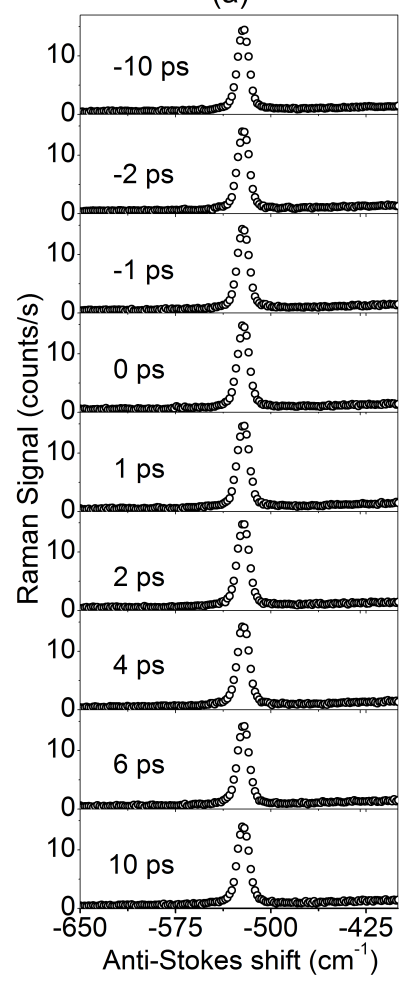

(b)

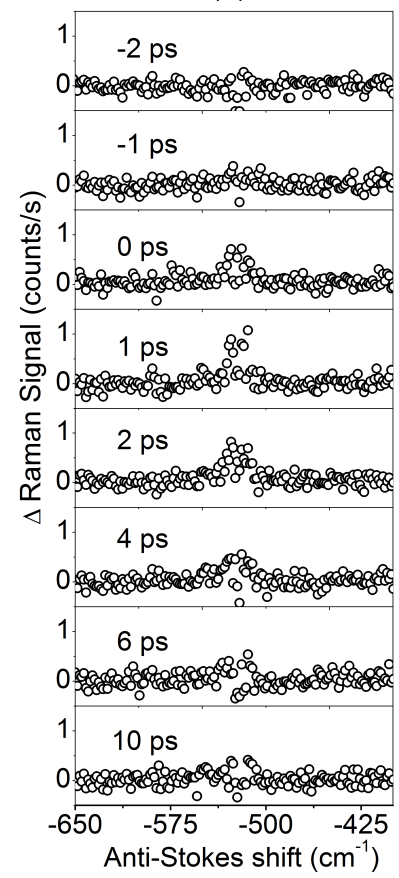

(a)
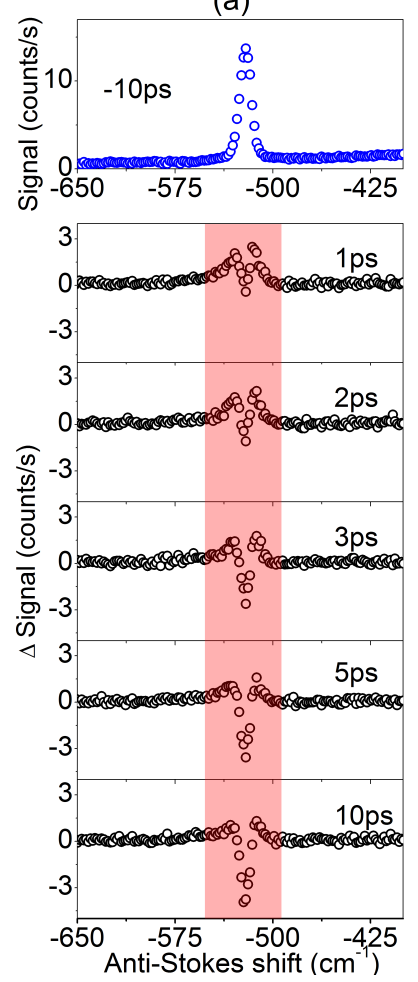

(b)
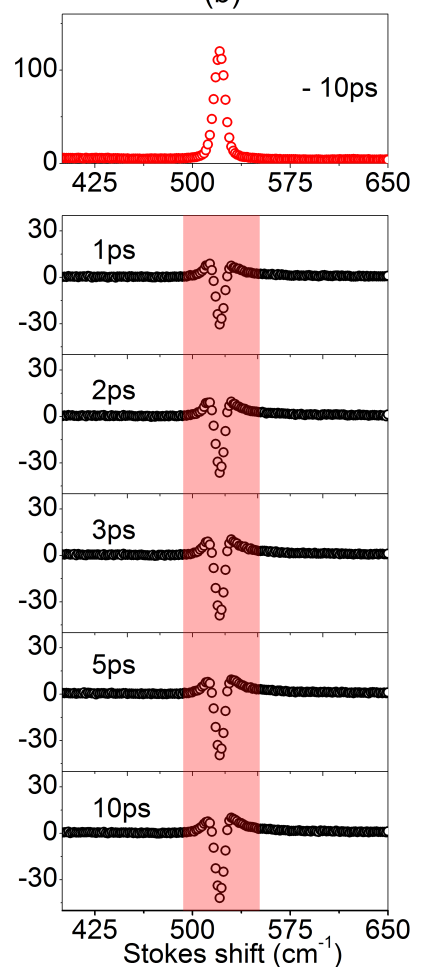

Figure 1: Time-resolved spontaneous silicon anti-Stokes Raman spectra recorded at a carrier density of $\sim 1.8 \times 10^{18} \mathrm{~cm}^{-3}$. (a) Raman spectra at various delay times before and after optical excitation. (b) Difference spectra obtained by subtracting the spectra at $-10 \mathrm{ps}$ from the spectra in (a).

pling of the optical phonon to this continuum leads to a pronounced Fano asymmetry ${ }^{41 / 42}$ of the optical phonon Raman response. These coupled spectral features lead to an intricate time-domain evolution following optical excitation, $\frac{17 / 43}{17}$ obscuring the pure population dynamics. As we will see, however, the detection of both the Stokes and anti-Stokes responses allows for the determination of the transient optical phonon population dynamics, while simultaneously yielding information on the temporal-evolution of the Raman scattering efficiency. Interestingly, the optically induced valence band hole density is found to lead to a transient enhancement of the Fano effect which can be observed in real time.

Figure 1 shows the transient anti-Stokes Raman spectra of silicon with a photoexcited carrier density of around $\sim 1.8 \times 10^{18} \mathrm{~cm}^{-3}$ measured at various pumpprobe delay times. ${ }^{44}$ For this relatively low transient carrier density, one observes a fast increase of the intensity near the optical phonon response at $-520 \mathrm{~cm}^{-1}$ which subsequently decreases with time until it vanishes after 6 - $10 \mathrm{ps}$. This corresponds to the expected creation of an optical phonon population through the electronphonon interaction, which subsequently decays on a ps timescale through anharmonic coupling with acoustic phonons $26127 / 29130145$

However, this simple picture is incomplete, as demon-

Figure 2: Lower panels: Time-resolved spontaneous silicon Raman difference spectra recorded at a carrier density of $\sim 1.8 \times 10^{19} \mathrm{~cm}^{-3}$ for anti-Stokes (a) and Stokes (b) scattering. These spectra are obtained by subtracting the spectra recorded for $-10 \mathrm{ps}$ delay (top panels) from the spectra recorded at positive pump-probe delays.

strated in Fig. 2 at higher excited carrier density $(\sim 1.8 \times$ $10^{19} \mathrm{~cm}^{-3}$ ) from both anti-Stokes (left panels) and Stokes (right panel) difference Raman spectra. Particularly striking is the non-trivial substructure and the notable asymmetry between the Stokes and anti-Stokes spectra. At early delay times (1-2 ps) the anti-Stokes difference spectra show a positive response near the phonon frequency, with slight negative difference signal in the middle, while a negative difference signal dominates on the Stokes side. For longer time delays, the negative signal on both sides gains strength until the Stokes and antiStokes signals become symmetric with a negative component in the middle and positive shoulders on the sides at $\sim 10 \mathrm{ps}$. The negative phonon difference signal persists for several nanoseconds, which is on the order of the carrier population lifetime. These spectral features originate from mixed effects of the optically induced phonon and hole populations, spectral broadening, and a Fano interference with the hole continuum. ${ }^{43}$ We first focus on the observed changes in the overall signal intensity.

The spectra from Fig. 2 were integrated over the optical phonon contribution $\left(490 \mathrm{~cm}^{-1}\right.$ to $\left.550 \mathrm{~cm}^{-1}\right)$ and corrected by subtracting the increasing electronic background ${ }^{46}$ The resulting relaxation dynamics are shown in Fig. 3a. The Stokes signal shows an immedi- 
(b)
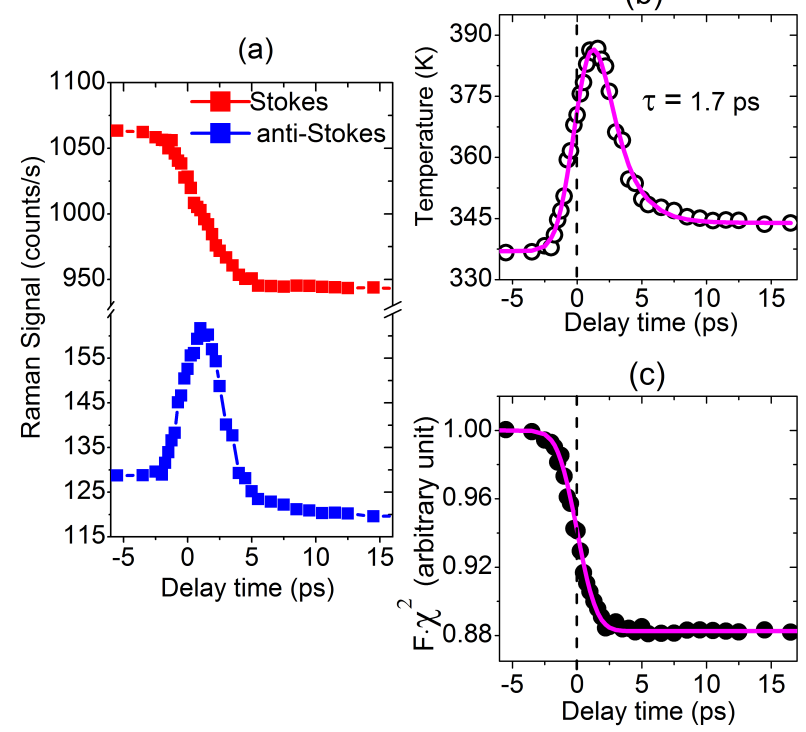

Figure 3: Relaxation dynamics of phonon equivalent temperature and Raman scattering strength at a carrier density of $\sim 1.8 \times 10^{19} \mathrm{~cm}^{-3}$. (a) Decay of the optical phonon intensity for Stokes scattering (red squares) and anti-Stokes scattering (blue squares). (b) Extracted phonon temperature evolution. (c) Time-evolution of the Raman scattering strength $F \cdot \chi^{2}$.

ate decrease in the scattering intensity after optical excitation. In contrast, the anti-Stokes signal first shows a rapid increase of scattering intensity which decreases only at later time delays. To understand this behavior, we note that the Raman signal for a specific phonon mode is determined by the population $n_{p}$, the Raman tensor $\chi^{2}$, and the related optical coefficient function $F$. It can be expressed as

$$
\mathrm{I}_{\mathrm{S}}=C \cdot F_{S} \cdot \omega_{S}^{3} \cdot \chi_{S}^{2} \cdot\left(1+n_{p}\right)
$$

and

$$
\mathrm{I}_{\mathrm{AS}}=C \cdot F_{A S} \cdot \omega_{A S}^{3} \cdot \chi_{A S}^{2} \cdot n_{p}
$$

for the Stokes and anti-Stokes response, respectively. 34 The factor $C$ includes the factors which are equal for both sides, such as the excitation laser energy dependence and the excitation being scattered from (i.e. the phonon frequency). The optical coefficient function $F$ can be written as

$$
F_{S}=\frac{\mathcal{T}_{S}}{\left(\alpha_{L}+\alpha_{S}\right) \cdot \eta_{S}}
$$

and

$$
F_{A S}=\frac{\mathcal{T}_{A S}}{\left(\alpha_{L}+\alpha_{A S}\right) \cdot \eta_{A S}}
$$

for Stokes and anti-Stokes scattering, respectively. Here $\mathcal{T}, \alpha$ and $\eta$ are the transmission coefficient, absorption coefficient and refractive index at the corresponding photon energies. These optical constants and the Raman tensor are strongly resonance dependent, and usually depend on temperature, which complicates the determination of phonon population based on the above formula. However, as demonstrated in Ref. 34, these correction factors are close to unity well below the resonance energy $(3.45 \mathrm{eV})$. As such, the following relation holds:

$$
F_{\mathrm{S}} \cdot \chi_{\mathrm{S}}^{2} \approx F_{\mathrm{AS}} \cdot \chi_{\mathrm{AS}}^{2} \equiv F \cdot \chi^{2}
$$

Using this relation and formula (1) and (2), one can now determine the phonon population and the phonon temperature from the experimental data. The resulting phonon temperature and the scattering strength $F \cdot \chi^{2}$ are plotted in Fig. 3b and 3c. Before time zero, the phonon temperature is $\sim 340 \mathrm{~K}$, showing an average heating above room temperature. Upon excitation, the phonon temperature rapidly increases to $\sim 390 \mathrm{~K}$ after which it decays close to the average value within a few picoseconds. An exponential fit gives a lifetime of approximately 1.7 ps (solid line in Fig.3p), consistent with the expected decay dynamics of optical phonons into acoustic phonons $13 / 17 / 29 \mid 45$

More surprising is the rapid decrease of about $10 \%$ in the scattering strength $F \cdot \chi^{2}$ upon excitation. A convolution of a step function and a Gaussian with half width of our setup resolution fits well (solid line in Fig. 3c) to the curve. Apparently, the reduction of the scattering strength can either originate from changes in the optical coefficient function $F$ (which is dictated by the optical constants), or from the Raman tensor square $\chi^{2}$ immediately after photoexcitation. Generally, the change of optical constants upon excitation are small. To confirm this, we performed a reflectivity measurement with identical excitation conditions, which shows that the changes of reflectivity upon excitation is less than $0.2 \% \underline{47}$ This small change in reflectivity, indicating a negligible change of the refractive index and thus the other derived optical constants, is not sufficient to explain the observed large change in scattering strength $F \cdot \chi^{2}$.

A more likely scenario for the observed decrease in scattering strength $F \cdot \chi^{2}$ arises from the resonant nature of the Raman scattering in silicon at the Raman probe excitation energies used in this study. The scattering is resonant to vertical transitions from the topmost valence band to the lowest conduction band in the $\Gamma-L$ direction in the Brillouin zone, and is thereby proportional to charge population! $\frac{48}{}$ In addition to this, excitonic effects are thought to play a role as well. $\frac{49}{}$ The pump laser pulse optically induces a hole density in the valence band, as evidenced by the observed changes in the hole continuum scattering (Fig. $4 \mathrm{~d}$ ) $\stackrel{46}{ }^{46}$ The optically induced charge density could lead to changes in the excitonic effects on the Raman resonance discussed in Ref. 49, which would lead to a strongly probe pulse energy dependent change 
(a)

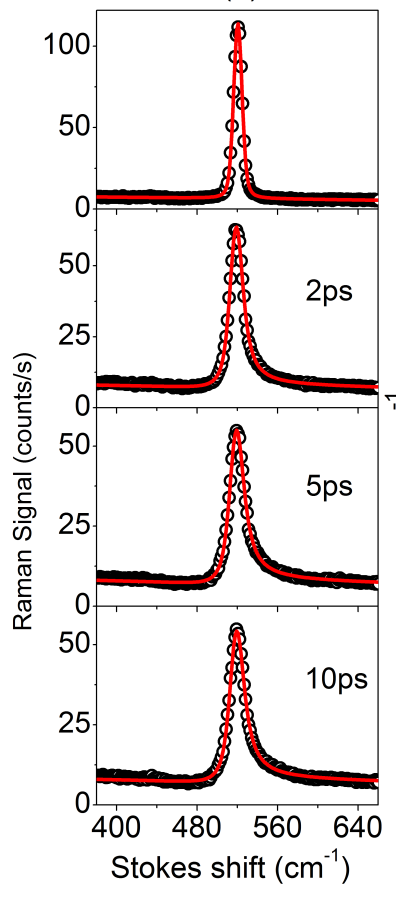

(b)

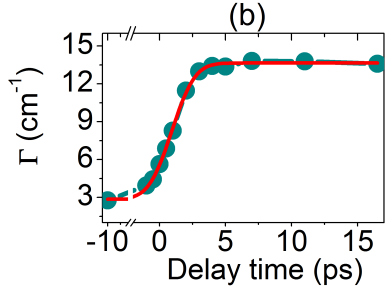

(c)
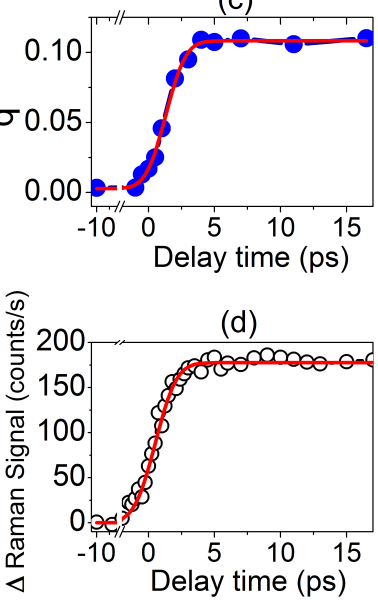

Figure 4: Transient Fano interference and hole population dynamics at a photoinduced density of $\sim 4.3 \times 10^{19} \mathrm{~cm}^{-3}$. (a) Time dependent Raman spectra (circles) and Fano-fits (red lines). (b) Fitted line-width parameter $\Gamma$. (c) Fitted inverse Fano parameter $q^{-1}$. (d) Hole scattering dynamics integrated in the spectral range from $650 \mathrm{~cm}^{-1}$ to $750 \mathrm{~cm}^{-1}$.

in $\chi^{2}$. However, our experiments show that the optically induced changes in the Raman spectra do not depend on probe energy within our experimental accuracy $[50$ Excitonic effects therefore do not appear to play a large role in the tensor quench. Obviously the resonant enhancement also strongly depends on the number of available initial and final states. Here, in particular the hole population in the vicinity of the $\Gamma$-point is of interest ${ }^{[48}$ Recent quantum theoretical work on silicon has shown that a few percent of the valence band states will be unoccupied at a photoexcitation density of $\sim 10^{19} \mathrm{~cm}^{-3}$, (Ref.51) which would lead to a reduction of $\chi^{2}$ of about $10 \%$ (Refs. 52 and (53). This is in good agreement with our observations, and we hence attribute the transient reduction of the Raman resonance to the photoinduced hole density.

We now turn to the line shape of the phonon response, and hole dynamics. Fano interference between the optical phonon and the electronic hole continuum in $p$-doped silicon is expected to be weak for the $512 \mathrm{~nm}$ probe wavelength. $37 / 38 / 40]$ However, at higher excitation densities, the changes in the Fano interference induced by the optical excitation are observable, $\frac{43}{3}$ as can be seen in Fig.4a, which shows the transient Raman Stokes spec-

tra (circles) at an excitation density of $\sim 4.3 \times 10^{19} \mathrm{~cm}^{-3}$ for various time delays. Generally the Fano line-shape can be described by

$$
\mathrm{I} \propto \frac{(q+\epsilon)^{2}}{1+\epsilon^{2}}
$$

where $q$ is the so-called Fano parameter which is inversely proportional to the spectral density of the electronic continuum, and $\epsilon=2\left(\omega-\omega_{0}\right) / \Gamma$ with $\omega_{0}$ the bare phonon frequency, and $\Gamma$ the line width parameter, which apart from the bare phonon part, has a contribution due to the Fano interference proportional to the spectral density of the electronic continuum. ${ }^{54}$ The transient behavior of the inverse Fano parameter $q^{-1}$ and the line-width $\Gamma$ obtained from fits (solid lines in Fig. 4a) are depicted in Fig. $4 \mathrm{~b}$ and 4 c. Before time zero, the Raman peak resembles a Lorentzian line shape $\left(q^{-1} \approx 0.003\right)$, whereas after photoexcitation the line-shape shows a pronounced Fano asymmetry $\left(q^{-1} \approx 0.11\right)$. As expected, a nearly stepwise time scale of the initial response of both $\Gamma$ and $q^{-1}$ were observed, which are identical to the electronic continuum dynamics (Fig. $4 \mathrm{~d}$ ). Since these parameters are all governed by the dynamics of the optically induced long-lived holes, no decay of these induced effects is observed within the measured time window. Finally, we note the values of the Fano parameters in our pump-probe experiments of pure silicon are consistent with results obtained from steady-state Raman scattering silicon for similar hole-

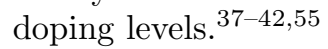

In summary, time-resolved spontaneous Raman scattering experiments on an intrinsic semiconductor show a prominent dynamic asymmetry between both the intensity and line-shape of the phononic Stokes and antiStokes responses. These asymmetries originate from the interplay between the transient phonon population dynamics, a quench of the phonon Raman resonance due to a photoinduced hole population, and an enhanced Fano interference due to the same hole population, in line with simple expectations. The present results provide a deeper understanding of the spontaneous Raman-scattering response of semiconductors to strong optical excitation, and expand the use of of time-resolved spontaneous Raman scattering in the study of collective excitation and quasiparticle dynamics in solid state materials.

This project was partially financed by the Deutsche Forschungsgemeinschaft (DFG) through SFB-1238 project B05, and instrument grant INST217/782-1. We gratefully acknowledge fruitful discussions with Daniele Fausti. R. B. V. acknowledges partial funding through the Bonn-Cologne Graduate School of Physics and Astronomy (BCGS).

J. Z. and R. B. V. contributed equally to this work. 
* Corresponding author pvl@ph2.uni-koeln.de

1 P. Bhattacharya, R. Fornari, and H. Kamimura, Comprehensive semiconductor science and technology, (Elsevier, Boston, 2011).

${ }^{2}$ M. Hase, M. Kitajima, A. M. Constantinescu, and H. Petek, The birth of a quasiparticle in silicon observed in timefrequency space, Nature 426, 51 (2003).

${ }^{3}$ M. Hase and M. Kitajima, Interaction of coherent phonons with defects and elementary excitations, J. Phys. Condens. Matter 22, (2010).

4 A. Othonos, Probing ultrafast carrier and phonon dynamics in semiconductors, J. Appl. Phys. 83, 1789 (1998).

5 A. J. Sabbah and D. M. Riffe. Measurement of silicon surface recombination velocity using ultrafast pumpprobe reflectivity in the near infrared, J. Appl. Phys. 88, 6954 (2000).

6 A. J. Sabbah and D. M. Riffe. Femtosecond pump-probe reflectivity study of silicon carrier dynamics, Phys. Rev. B 66, 165217 (2002).

7 D. M. Riffe and A. J. Sabbah, Coherent excitation of the optic phonon in Si: Transiently stimulated Raman scattering with a finite-lifetime electronic excitation, Phys. Rev. B 76, 085207 (2007).

${ }^{8}$ K. Kato, K. Oguri, A. Ishizawa, K. Tateno, T. Tawara, H. Gotoh, M. Kitajima, H. Nakano, and T. Sogawa, Doping-type dependence of phonon dephasing dynamics in Si, Appl. Phys. Lett. 98, 141904 (2011).

9 M. Trigo, M. Fuchs, J. Chen, M. P. Jiang, M. Cammarata, S. Fahy, D. M. Fritz, K. Gaffney, S. Ghimire, A. Higginbotham, S. L. Johnson, M. E. Kozina, J. Larsson, H. Lemke, A. M. Lindenberg, G. Ndabashimiye, F. Quirin, K. Sokolowski-Tinten, C. Uher, G. Wang, J. S. Wark, D. Zhu, and D. A. Reis, Fourier-transform inelastic x-ray scattering from time-and momentum-dependent phononphonon correlations, Nat. Phys. 9, 790 (2013).

10 D. C. Hannah, K. E. Brown, R. M. Young, M. R. Wasielewski, G. C. Schatz, D. T. Co, and R. D. Schaller, Direct measurement of lattice dynamics and optical phonon excitation in semiconductor nanocrystals using femtosecond stimulated Raman spectroscopy, Phys. Rev. Lett. 111, 107401 (2013).

11 D. Fausti and P. H. M. van Loosdrecht, in Chapter 14 of Optical Techniques for Solid-State Materials Characterization, edited by R. P. Prasankumar and A. J. Taylor, (CRC Press, Boca Raton London New York, 2012).

12 R. B. Versteeg, J. Zhu, P. Padmanabhan, C. Boguschewski, R. German, M. Goedecke, P. Becker, and P. H. M. van Loosdrecht, A tunable time-resolved spontaneous Raman spectroscopy setup for probing ultrafast collective excitation and quasiparticle dynamics in quantum materials, Struct. Dyn. 5, 044301 (2018).

13 J. A. Kash, J. C. Tsang, and J. M. Hvam. Subpicosecond time-resolved Raman spectroscopy of LO phonons in GaAs, Phys. Rev. Lett. 54, 2151 (1985).

14 D. Y. Oberli, D. R. Wake, M. V. Klein, J. Klem, T. Henderson, and H. Morkoç, Time-resolved Raman scattering in GaAs quantum wells, Phys. Rev. Lett. 59, 696 (1987).

15 K. T. Tsen and H. Morkoç, Population relaxation time of nonequilibrium lo phonons and electron-phonon interactions in $\mathrm{GaAs}-\mathrm{Al}_{x} \mathrm{Ga}_{1-x}$ As multiple-quantum-well structures, Phys. Rev. B 34, 4412 (1986).
${ }^{16}$ K. T. Tsen and H. Morkoç, Subpicosecond timeresolved Raman spectroscopy of LO phonons in GaAs $\mathrm{Al}_{x} \mathrm{Ga}_{1-x}$ As multiple-quantum-well structures, Phys. Rev. B 38, 5615 (1988).

17 J. J. Letcher, K. Kang, D. G. Cahill, and D. D. Dlott, Effects of high carrier densities on phonon and carrier lifetimes in Si by time-resolved anti-stokes Raman scattering, Appl. Phys. Lett. 90, 252104 (2007).

18 K. T. Tsen, J. G. Kiang, D. K. Ferry, and H. Morkoç, Subpicosecond time-resolved Raman studies of LO phonons in GaN: Dependence on photoexcited carrier density, Appl. Phys. Lett. 89, 112111 (2006).

19 A. L. Malinovsky, A. A. Makarov, and E. A. Ryabov, Slow intramolecular vibrational redistribution: the latest results for trifluoropropyne, a comparison with the other terminal acetylenes and the mechanism, Phys. Scripta 85, 058102 (2012).

20 K. Iwata, H. Okajima, S. Saha, and H. O. Hamaguchi, Local structure formation in alkyl-imidazolium-based ionic liquids as revealed by linear and nonlinear Raman spectroscopy, Acc. Chem. Res. 40, 1174 (2007).

21 S. K. Sahoo, S. Umapathy, and A. W. Parker, Timeresolved resonance Raman spectroscopy: Exploring reactive intermediates, Appl. Spectrosc. 65, 1087 (2011).

22 A. Matsuda, H. Nagao, K. G. Nakamura, and K. Kondo, Time-resolved Raman spectra of ring-stretching modes of benzene derivatives under laser-driven shock compression at $1 \mathrm{GPa}$, Chem. Phys. Lett. 372, 911 (2003).

23 I. E. I. Petterson, F. W. L. Esmonde-White, W. de Wilde, M. D. Morris, and F. Ariese, Tissue phantoms to compare spatial and temporal offset modes of deep Raman spectroscopy, Analyst 140, 2504 (2015).

${ }^{24} \mathrm{H}$. Hamaguchi and T. L. Gustafson, Ultrafast timeresolved spontaneous and coherent Raman spectroscopy: the structure and dynamics of photogenerated transient species, Annu. Rev. Phys. Chem. 45, 593 (1994).

25 S. G. Kruglik, J.-C. Lambry, J.-L. Martin, M. H. Vos, and M. Negrerie, Sub-picosecond raman spectrometer for timeresolved studies of structural dynamics in heme proteins, J. Raman Spectrosc. 42, 265 (2011).

26 K. Kang, T. Ozel, D. G. Cahill, and M. Shim, Optical phonon lifetimes in single-walled carbon nanotubes by time-resolved Raman scattering, Nano Lett., 8, 4642 (2008).

27 D. Song, F. Wang, G. Dukovic, M. Zheng, E. D. Semke, L. E. Brus, and T. F. Heinz, Direct measurement of the lifetime of optical phonons in single-walled carbon nanotubes, Phys. Rev. Lett. 100, 225503 (2008).

${ }^{28}$ H. Yan, D. Song, K. F. Mak, I. Chatzakis, J. Maultzsch, and T. F. Heinz, Time-resolved Raman spectroscopy of optical phonons in graphite: Phonon anharmonic coupling and anomalous stiffening, Phys. Rev. B 80, 121403 (2009).

29 K. Kang, D. Abdula, D. G. Cahill, and M. Shim, Lifetimes of optical phonons in graphene and graphite by timeresolved incoherent anti-Stokes Raman scattering, Phys. Rev. B 81, 165405 (2010).

30 I. Chatzakis, H. Yan, D. Song, S. Berciaud, and T. F. Heinz, Temperature dependence of the anharmonic decay of optical phonons in carbon nanotubes and graphite, Phys. Rev. B 83, 205411 (2011).

31 J.-A. Yang, S. Parham, D. Dessau, and D. Reznik, Novel 
electron-phonon relaxation pathway in graphite revealed by time-resolved Raman scattering and angle-resolved photoemission spectroscopy, Sci. Rep. 7, 40876 (2017).

32 J. Zhu, R. German, B. Senkovskiy, D. Haberer, F. Fischer, A. Grüneis, and P. H. M. van Loosdrecht, Exciton and phonon dynamics in highly aligned 7-atom wide armchair graphene nanoribbons as seen by time-resolved spontaneous Raman scattering, Nanoscale 10, 17975 (2018).

33 R. Loudon, Time-reversal symmetry in light-scattering theory, J. Raman Spectrosc. 7, 10 (1978).

34 A. Compaan and H. J. Trodahl, Resonance Raman scattering in Si at elevated temperatures, Phys. Rev. B 29, 793 (1984).

35 D. Fausti, O. V. Misochko, and P. H. M. van Loosdrecht, Ultrafast photoinduced structure phase transition in antimony single crystals, Phys. Rev. B 80, 161207 (2009).

36 R. P. Saichu, I. Mahns, A. Goos, S. Binder, P. May, S. G. Singer, B. Schulz, A. Rusydi, J. Unterhinninghofen, D. Manske, P. Guptasarma, M. S. Williamsen, and M. Rübhausen, Two-component dynamics of the order parameter of high temperature $\mathrm{Bi}_{2} \mathrm{Sr}_{2} \mathrm{CaCu}_{2} \mathrm{O}_{8+\delta}$ superconductors revealed by time-resolved Raman scattering, Phys. Rev. Lett. 102, 177004 (2009).

37 F. Cerdeira, T. A. Fjeldly, and M. Cardona, Interaction between electronic and vibronic Raman scattering in heavily doped silicon, Solid State Commun. 13, 325 (1973).

38 F. Cerdeira, T. A. Fjeldly, and M. Cardona, Effect of free carriers on zone-center vibrational modes in heavily doped p-type Si. II. optical modes, Phys. Rev. B 8, 4734 (1973).

39 M. Chandrasekhar, J. B. Renucci, and M. Cardona, Effects of interband excitations on Raman phonons in heavily doped $n$ Si, Phys. Rev. B 17, 1623 (1978).

40 B. G. Burke, J. Chan, K. A. Williams, Z. L. Wu, A. A. Puretzky, and D. B. Geohegan, Raman study of Fano interference in $p$-type doped silicon, J. Raman Spectrosc. 41, 1759 (2010).

41 U. Fano, Effects of configuration interaction on intensities and phase shifts, Phys. Rev. 124, 1866 (1961).

42 U. Fano, G. Pupillo, A. Zannoni, C. W. Clark, On the absorption spectrum of noble gases at the arc spectrum limit, J. Res. Natl. Inst. Stan. 110, 583 (2005).

43 K. Kato, Y. Hasegawa, K. Oguri, T. Tawara, T. Nishikawa, and H. Gotoh, Relationship between the Fano asymmetry parameter and time-domain spectra studied by timeresolved measurement of carriers and phonons in $p$-type $\mathrm{Si}$, Phys. Rev. B 97, 104301 (2018).

44 The carrier density has been determined from the incident photon density and the optical properties of silicon, see Supplemental Material.

45 P. G. Klemens, Anharmonic decay of optical phonons, Phys. Rev. 148 (1966).

${ }^{46}$ K. Tanaka, H. Ohtake, and T. Suemoto, Determination of intervalley scattering time in germanium by subpicosecond time-resolved Raman spectroscopy, Phys. Rev. Lett. 71, 1935 (1993).

47 See Supplemental Material for the transient reflectivity measurement.

48 J. B. Renucci, R. N. Tyte, and M. Cardona, Resonant Raman scattering in silicon, Phys. Rev. B 11, 3885 (1975)

49 Y. Gillet and M. Giantomassi, and X. Gonze, Firstprinciples study of excitonic effects in Raman intensities, Phys. Rev. B 88, 094305 (2013).

50 See Supplemental Material for transients at $450 \mathrm{~nm}$ Raman probe wavelength.

51 D. Sangalli, S. D. Conte, C. Manzoni, G. Cerullo, and A. Marini, Nonequilibrium optical properties in semiconductors from first principles: A combined theoretical and experimental study of bulk silicon, Phys. Rev. B 93, 195205 (2016).

52 See Supplemental Material for the two-level model which relates the Raman tensor reduction to the photoinduced hole density.

${ }^{53}$ R. W. Boyd, Nonlinear Optics, $3^{\text {rd }}$ ed. (Elsevier, Amsterdam, 2008).

54 M. Cardona and G. Güntherodt, Light Scattering in Solids $I V$, in page 128 (Springer-Verlag, Berlin, Heidelberg, New York, Tokyo, 1984).

55 See Supplemental Material for the data of the transient versus steady-state Fano parameter comparison. 